\title{
3D Vessel Modelling and Experimental Platform of Blood Flow
}

\author{
Irina Andra TACHE*, Severus Constantin OLTEANU \\ University Politehnica of Bucharest, 313 Splaiul Independenței, Bucharest, Romania \\ irina.tache@acse.pub.ro (*Corresponding author) \\ severus.olteanu@acse.pub.ro
}

\begin{abstract}
The 3D reconstruction of the vessels from two monoplane angiographic views is used to build patient-specific vessel model. For this study, the data was acquired from seven patients suffering from ischemic diseases. The results were validated in comparison with the vessel lengths computed with the dedicated software installed on the angiograph. Also, a blood flow simulator based on feedback control loop was designed and tested.
\end{abstract}

Keywords: 3D reconstruction, Proportional control, Monoplanar angiography.

\section{Introduction}

The detection of vascular abnormal structures is becoming more frequent in clinics because of the stressful way of living, aging of population and eating habits.

A lot of human and material resources are employed in order to eliminate or diminish the pain and still the diagnosis and treatment are not accurate because they lack the hemodynamic measures.

Therefore, the interest in improving the vascular diseases diagnosis and treatment is very high. The most recent techniques, devices and algorithms are helping the medical staff develop better medical imaging acquisition and processing.

Nowadays with the growing of the industry of super-computers, the simulation based on computation fluid dynamics is used more frequently in recreating the physiological conditions of blood flow. Still, due to complex modelling of fluid structure interactions, it is a time-consuming technique.

The most important parameters for identifying the cardiovascular diseases are the blood flow disorders correlated with changes in morphology. The in vivo measurements for some arteries with large diameter are possible with a catheter on whose tip a flow transducer is installed.

Still, the construction of a platform for focussing on the different blood flow patterns throughout the vascular structures will be welcomed in the present context.

The above-mentioned device has the advantage of particularizing a certain vessel which can be normal or abnormal, such as the ones affected by aneurism or stenosis. It can be used in case that computational simulation is not effective.

The main problem will be the reproduction of the whole vascular structure which could take on the real properties of the vessels, such as elasticity, morphology and the whole physiological fluid dynamics function.

The building of the so-called, patient-specific vascular anatomic model is based on a real image set collected from the patient. A prototype can be further created by means of the $3 \mathrm{D}$ printers.

Nevertheless, the process of creating the 3D vascular model from medical images as an accurate $3 \mathrm{D}$ printing is a challenging work as it is revealed and explained in detail in (Ioniță et al., 2014). The authors have used a 3D printer based on polyjet technique with a resolution of 16 micron to print complex vasculature using standard and flexible materials.

Wächter (2009) proposes a platform to simulate the blood circulation through cerebral arteries, especially for the Willis circle region, in order to validate an algorithm for hemodynamic parameters estimation. This equipment doesn't include a control algorithm; it is simply meant to reproduce a pulsatile flow manually, by means of a continuous pump and a cylinder which modifies the flow depending on the cross sectional area of the main tubing. For the vessel phantom the author generated the model from the $3 \mathrm{D}$ reconstruction of blood vessels from rotational C-arm angiograph. A specialised company printed the $3 \mathrm{D}$ model using a bio-material.

In (Mechoor et al., 2016) the bench top test setup is a programmable pulsatile pump, suitable 
for reproducing physiologically realistic blood pressure and flow for analyzing the fluid mechanics of the circulatory system. Based on the experiments which have been carried out, it accurately reproduces localized physiological flow waveforms for abdominal aorta under exercise. The total cost of the prototype is more than 1000 euro.

In (Drost et al., 2018) a programmable pulsatile pump system was built using Arduino microcontroller to implement a feed forward signal computed in Matlab ${ }^{\circledR}$. It can reproduce a localized flow waveform. The total cost of the system excluding the sensor is 350 euro.

The papers (Mechoor et al., 2016, Drost et al., 2018) didn't use a pacient-specific vessel prototype for testing.

In the current study, the process of creating the $3 \mathrm{D}$ model based on coronary angiogram images is proposed with a view to building a low cost experimental platform for studying the blood flows.

The monoplane angiography is the gold standard for diagnosing the vascular diseases due to its good spatial resolution. However, for the 3D vessel reconstruction the rotational angiography or the Computed Tomography is generally preferred (Wächter, 2009; Ioniță et al., 2014) due to higher accuracy to represent the real vessel morphology.

In this paper the 3D model of the vessel is obtained from the monoplane angiography which is a more approximative approach. The most important 3D reconstruction parameters are optimised and the algorithm validation is presented.

The main limitation of the study is that $3 \mathrm{D}$ model was not printed due to the high quality requirements from the $3 \mathrm{D}$ printer in terms of resolution and flexible material.

The proposed platform for simulating the arterial blood flow circulation includes a feedback control loop and it consists in an Arduino microcontroller, a reservoir filled with saline water, the pump which delivers the desired flow waveform, a silicone tubular vessel phantom and the inline liquid flow meter.

The objective of this research is to develop a simple to implement and powerful enough architecture that can reproduce the arterial blood flow patterns with a total cost around 100 euro.
The vessel simulator can be used for medical device testing and physiological or functional evaluations as well as in case the computational simulations are not effective or sufficiently descriptive.

The paper is organised as following: in the section 2 there are described the data characteristics, the methods used for 3D reconstruction and the experimental platform construction, in the section 3 the results are presented and in section 4 the conclusions are drawn.

\section{Materials and Methods}

\subsection{Data from the patients}

The patients investigated in this study were suffering from ischemic diseases, with different degrees of myocardial ischemia.

The X-ray angiography is an invasive medical procedure which involves the insertion of a catheter into the femoral or radial artery which is guided to the heart vessels.

The $\mathrm{C}$-arm angiographic system consists of a detector and an X-ray source. The C-arm system is designed to rotate around the patient on two axes, one from patient's right arm to the left arm (primary angle) and another one from cranium to the caudal area (secondary angle).

The cardiac angiograms, created by means of a Siemens Axiom Artis Zee angiograph were acquired from the Clinical Emergency Hospital of Bucharest within the national grant entitled High Performance Computing of Personalized Cardio Component Models based on an ethical protocol signed with the patients.

The image data characteristics can be noticed in the Table 1 (Tache, 2015).

Table 1. Image characteristics (Tache, 2015)

\begin{tabular}{|l|l|}
\hline Parameters & Cardiac \\
\hline No. of images per series & 70 \\
\hline Spatial resolution & $512 * 512$ \\
\hline Temporal resolution & 15 images/second \\
\hline No. of bites stored per image & 8 \\
\hline
\end{tabular}

\subsection{Centerline detection}

This is an essential step in the 3D vessel reconstruction because it preserves its curvilinear shape and its length. 
The most important methods for accomplishing this operation are: the ridge detectors based on level set theory, morphological skeleton, contour pruning, fast matching and geometrical model based methods, region growing methods (Morar et al 2017b), filtering-based methods and vessels' tracking.

A simple graphical implementation is the manual selection of the intermediary pixels of the vessel centerline.

A graphical user interface developed in Matlab ${ }^{\circledR}$ enabled the user to select pixels on the medical image. The cubic spline function is used to interpolate these points and the output is plotted on the original image.

\subsection{Mathematical Modeling of the 3D Reconstruction}

The 3D reconstruction was intensively investigated ten years ago, simultaneously with the development of the digital medical imaging. There are many methods presented in the scientific literature for solving this problem, each one dedicated to a specific angiograph type, such as: the monoplane system (Shechter, 2004) where the coronary artery motion artefacts due to respiration are diminished, (Sprague et al., 2006) where more than two views are used to generate the 3D model, the biplane system (Bourantas et al., 2005), or the rotational system (Blondel et al., 2006) where the reconstruction is made from a single projection view, Garcia et al., 2007 where the optimal view is chosen avoiding vessel foreshortening effects.

All of these methods are time-consuming and the real time generation of the $3 \mathrm{D}$ model is tedious, but due to the algorithm parallelisation and cloud computing (Morar et al., 2017a, Petrescu et al., 2011; Marcu et al., 2017) the computational time is significantly improved.

The algorithm of 3D reconstruction from two uncalibrated angiographic views is based on (Brost et al., 2009 a, b).

The projection matrix $(\mathrm{P})$ defined as the $3 \mathrm{D}$ point mapping into the image points is computed as in eq. (1):

$$
P=\left[K_{i} \mid R_{p}\right]
$$

where $P \in R^{3 \times 4}, K_{i} \in R^{3 \times 3}$ is the camera intrinsic parameters' matrix, $R_{p} \in R^{3 \times 3}$ is the rotational matrix and $t \in R^{3}$ is the translation vector.
The 3D projection of the spatial point expressed in homogenous coordinates $\widetilde{p_{3 D}}=\left[p_{3 D}{ }^{T} 1\right]^{T}$ into the $2 \mathrm{D}$ point $\widetilde{p_{2 D}}$ is considered to be:

$\widetilde{p_{2 D}}=P \cdot \widetilde{p_{3 D}}$

Epipolar geometry also known as the geometry of stereo vision introduces the mathematical relations for the case when two cameras situated into different spatial locations view the same 3D scene. There is a $3 \mathrm{D}$ point on the vessel which has two 2D corresponding points projected into two angiographic views. The whole algorithm of finding the 3D points of the vessel centerline knowing the $2 \mathrm{D}$ corresponding points on two different image perspectives is described in (Tache, 2017).

An overview of the algorithm steps is:

a. Manual selection of the intermediary pixels of the vessel centerline in one image view

b. Tagging the 2D corresponding points of the vessel of interest from the second image view

c. Optimisation of some important reconstruction parameters

d. 3D reconstruction algorithm applied for the new parameters found at step c.

e. Generating the 3D model from the 3D vessel centerline obtained at step $\mathrm{d}$.

\subsection{Optimization of the Key Reconstruction Parameters}

Most of the 3D reconstruction parameters can be found among the metadata of the Dicom files of the image set. Still, there are errors due to the patient motion during the angiographic clinical investigation or because of the uncalibrated image acquisition.

In addition to the algorithm developed by (Brost et al., $2009 \mathrm{a}, \mathrm{b}$ ) an optimization step is included for completing the $3 \mathrm{D}$ reconstruction algorithm. It includes the following phases:

a. The initialization of the reconstruction parameters using the values extracted from the image metadata.

b. The construction of the cost function (O) adapted from (Yang et al. 2009) for minimisation the Euclidian distance between 
the given $2 \mathrm{D}$ vessel points into both images and the corresponding back projection points of the $3 \mathrm{D}$ reconstructed centerline pixels on those images. This is also known as the reconstruction error or the point transfer error, which it includes an additive operation on the spatial point thorough a translation vector describing the patient motion $\left(m_{t}\right)$ :

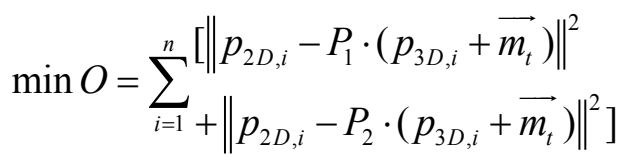

where $\mathrm{P}_{1}$ and $\mathrm{P}_{2}$ are the projection matrixes for both images.

c. The Levenberg - Marquant algorithm is used to compute the non-linear least square problem (Stefanoiu et al. 2014) generated by the cost function minimisation.

d. The 3D reconstruction algorithm is applied for the new values of the 11 optimised parameters computed in the previous step.

\subsection{Building the blood vessel platform}

The vessel simulator is built based on the closed loop principle shown in Fig. 1.

The elements from Fig. 1 are:

- The control unit: microcontroller and Buck converter,

- Execution element: the geared pump,

- The sensor: the inline flow meter,

- The process: the vascular structure and its case which can be filled with water in order to simulate the real medium of a vessel.

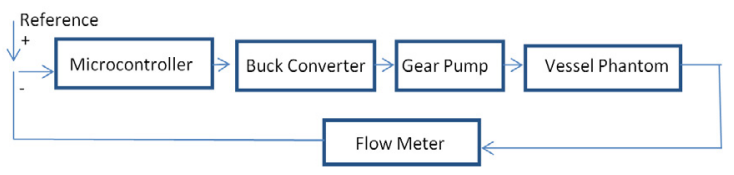

Figure 1. Closed loop flow circuit

An important step is setting the reference signal which is, in fact, the desired flow pattern and then the control algorithm for keeping the former throughout the functional cycle.

The numerical control signal is sent to the pump which, in response delivers the liquid with a certain flow into the vessel phantom equipped with a flow meter on its terminal.

\section{Results}

\subsection{D Reconstruction of vessel segment}

In order to exemplify the improvement brought by the $3 \mathrm{D}$ reconstruction optimisation step in the following, the $3 \mathrm{D}$ reconstruction of the left main coronary artery from a normal patient is presented below.

This example is provided to test the efficiency of the reconstruction algorithm when the correspondences between the points were settled with fewer matching errors, since both images appertain to the same cardiac cycle phase and a reference object (the catheter tip) was provided.

The Matlab ${ }^{\circledR}$ graphical user interface was used to implement the algorithm.

The corresponding points from the artery centerline were more easily tagged into different projection views, because it included the catheter tip where the contrast agent was injected.

Also, this vessel has fewer motions during the cardiac cycle and the selected images for the reconstruction were taken from the late diastole phase.

The imagistic expert selected some intermediate points of the centerline and its final coordinates were obtained based on the mathematical modelling of the segment through a cubic spline with a resolution of 100 fractions.

The reconstruction error is the image distance between the $2 \mathrm{D}$ points given by the user on the images and the 2D coordinates from the back projection of the spatial centerline points into those images. An optimisation algorithm was used in order to minimise this error.

There are also reconstruction parameters for each projection view such as, the distance between the source and detector and the distance between the source and isocenter - initialised with the distance from source to patient. All these parameters can adjust the magnification factor of the vessel on an image and the alpha (primary) and beta (secondary) angles. Some other general parameters are: the coordinates of the image plane center and the motion translation vector with the coordinates [mx, my, mz].

The optimisation is done by varying all three components of the motion vector. The results are shown in Table 2. The user centerline selection is 
Table 2. 3D Reconstruction of the vessel's centerline

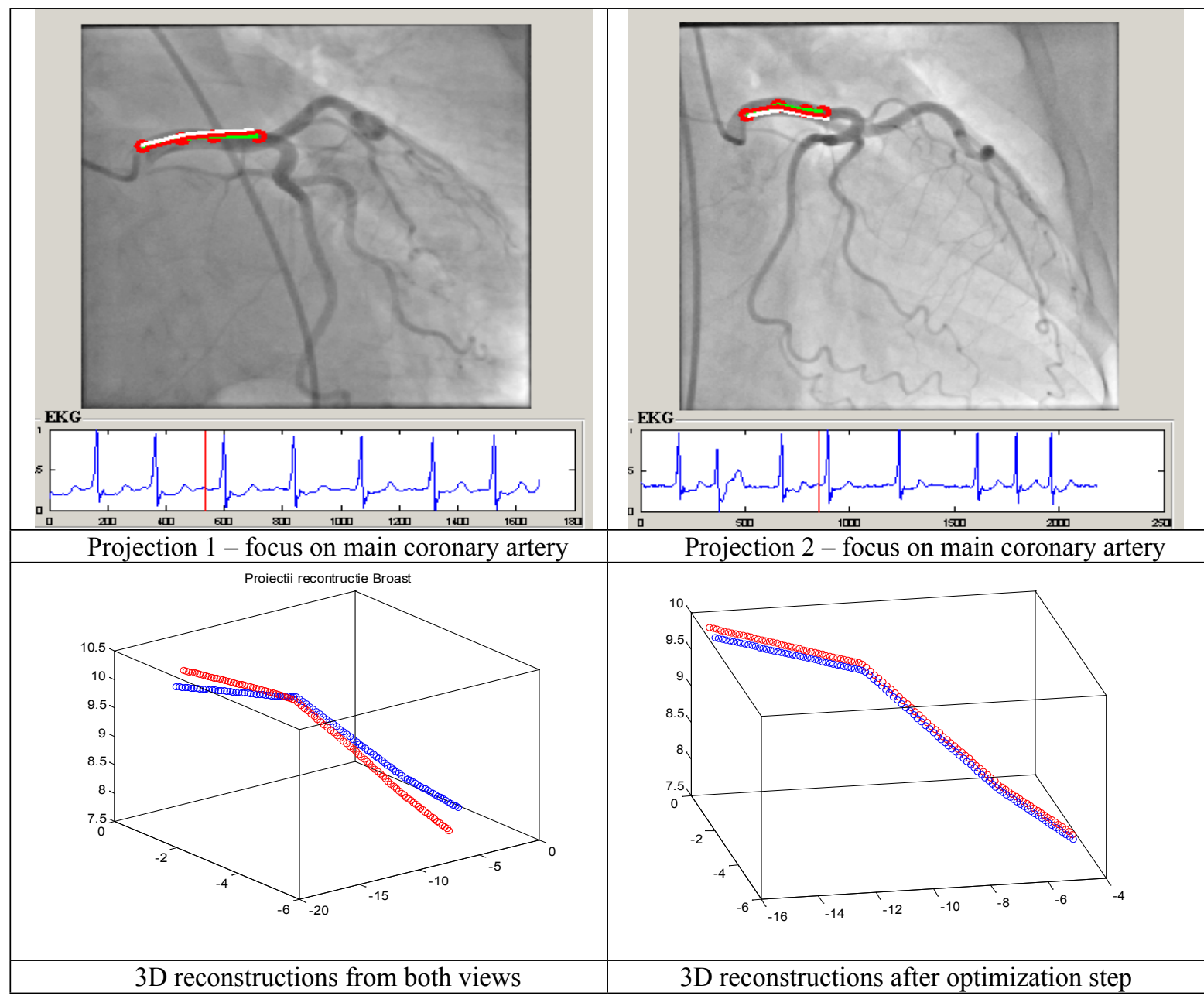

represented in green, the back projection for the initial reconstruction in white and the optimisation step in red.

The errors for the optimisation of all three coordinates of the vector describing the table motion on which the patient sits are lower than the initial reconstruction error without optimization (a mean of $1.8 \mathrm{~mm}$ from the Table 3 ).

Table 3. Quantification of the optimisation process

\begin{tabular}{|c|c|c|}
\hline & View 1 & View 2 \\
\cline { 2 - 3 } & $\begin{array}{c}\text { Recons. Error } \\
{[\mathrm{mm}]}\end{array}$ & $\begin{array}{c}\text { Recons. Error } \\
{[\mathrm{mm}]}\end{array}$ \\
\hline Initial & 1.66 & 1.95 \\
\hline Optimisation & 0.74 & 0.91 \\
\hline
\end{tabular}

According to medical literature, the length of the left main coronary artery is between 10 to $26 \mathrm{~mm}$. After the optimisation step, the vessel lengths were determined, as $24.4 \mathrm{~mm}$ for the first projection, $27 \mathrm{~mm}$ for the second one and $26 \mathrm{~mm}$ after the $3 \mathrm{D}$ reconstruction.
Only the 2D length from the second projection 2 overrode this range.

The algorithm sensitivity to a good matching of the $2 \mathrm{D}$ points of the vessel into both projections is therefore confirmed.

The algorithm was also tested for other six patients collected from the clinic. The data was previously analysed be means of a dedicated software installed on the angiographic equipment also known as the quantitative X-ray angiography software and the vessel segment was measured. The physician tagged the end points of the vessel on the image series. The algorithm used the epipolar geometry to detect the corresponding points of the vessel into the second view. The computed vessels' lengths were considered as ground truth data.

For each image set, the physician has tagged a certain vessel of interest, such as RCA - right coronary artery, LAD (left coronary artery), 
Table 4. Absolute vessels' lengths and the key location parameters of the C-arm system

\begin{tabular}{|c|c|c|c|c|c|c|}
\hline No Patient & \multicolumn{6}{|c|}{1} \\
\hline Analyzed Vessel & \multicolumn{2}{|l|}{ RCA } & \multicolumn{2}{|l|}{ LCX } & \multicolumn{2}{|c|}{ LAD post } \\
\hline Distance 3D $(\mathrm{cm})$ dedicated software & \multicolumn{2}{|l|}{3.74} & \multicolumn{2}{|l|}{5.23} & \multicolumn{2}{|l|}{8.4} \\
\hline \multirow[t]{2}{*}{ Distance 3D $(\mathrm{cm})$ proposed algorithm } & \multicolumn{2}{|l|}{4.28} & \multicolumn{2}{|l|}{4.81} & \multicolumn{2}{|l|}{7.9} \\
\hline & 1st View & 2nd View & 1st View & 2nd View & 1st View & 2nd View \\
\hline Positioner Primary Angle (degree) & 1.395 & -30.5 & -0.7 & 47 & -17.7 & -18 \\
\hline Positioner Secondary Angle (degree) & 0.9 & 1 & -40.1 & -20.2 & 28.4 & -15.6 \\
\hline Estimated Radiographic Magnification Factor & 1.392 & 1.397 & 1.595 & 1.389 & 1.457 & 1.448 \\
\hline No Patient & \multicolumn{2}{|c|}{2} & \multicolumn{2}{|c|}{3} & \multicolumn{2}{|c|}{4} \\
\hline Analyzed Vessel & \multicolumn{2}{|l|}{ LAD } & \multicolumn{2}{|l|}{ RCA } & \multicolumn{2}{|l|}{ RCA } \\
\hline Distance 3D $(\mathrm{cm})$ dedicated software & \multicolumn{2}{|l|}{2.39} & \multicolumn{2}{|l|}{7.5} & \multicolumn{2}{|l|}{9.54} \\
\hline \multirow[t]{2}{*}{ Distance 3D $(\mathrm{cm})$ proposed algorithm } & \multicolumn{2}{|l|}{2.85} & \multicolumn{2}{|l|}{8.13} & \multicolumn{2}{|l|}{9.88} \\
\hline & 1st View & 2nd View & 1st View & 2nd View & 1st View & 2nd View \\
\hline Positioner Primary Angle (degree) & 42.6 & -14.8 & 28.1 & -32.9 & 21 & -38.8 \\
\hline Positioner Secondary Angle (degree) & -36 & 41.5 & -2.22 & -2.2 & 0.2 & -14.9 \\
\hline Estimated Radiographic Magnification Factor & 1.493 & 1.509 & 1.353 & 1.422 & 1.252 & 1.391 \\
\hline No. Patient & \multicolumn{2}{|c|}{5} & \multicolumn{4}{|c|}{6} \\
\hline Analyzed Vessel & \multicolumn{2}{|l|}{ LAD } & \multicolumn{2}{|l|}{ LAD } & \multicolumn{2}{|l|}{ LAD } \\
\hline Distance 3D $(\mathrm{cm})$ dedicated software & \multicolumn{2}{|l|}{3.24} & \multicolumn{2}{|l|}{3.42} & \multicolumn{2}{|l|}{3.24} \\
\hline \multirow[t]{2}{*}{ Distance 3D $(\mathrm{cm})$ proposed algorithm } & \multicolumn{2}{|l|}{3.16} & 3.94 & & 3.16 & \\
\hline & 1st View & 2nd View & 1st View & 2nd View & 1st View & 2nd View \\
\hline Positioner Primary Angle (degree) & 4.6 & -31.7 & 4.6 & -31.7 & 4.6 & -31.7 \\
\hline Positioner Secondary Angle (degree) & -43.9 & -6.6 & -43.9 & -6.6 & -43.9 & -6.6 \\
\hline Estimated Radiographic Magnification Factor & 1.589 & 1.314 & 1.589 & 1.314 & 1.589 & 1.314 \\
\hline
\end{tabular}

LAD post (left coronary artery after angioplasty procedure), LCX (left circumflex artery).

From the Table 4 it seems that for patient 1 RCA, patient 2 , patient 3 , patient 4 and patient $6 \mathrm{LAD}$ the algorithm tends to overestimate the ground truth by a mean difference of $0.498 \mathrm{~cm}$ between the vessels' lengths and for the rest tends to underestimate by a mean value of $-0.29 \mathrm{~cm}$.

The vessel length differences can be associated with the mismatching of the vessel points into the images.

\subsection{Blood vessel simulator}

The developed platform (Fig. 2) is an embedded mobile platform, where a microcontroller is controlling the dynamics of a pump via data stored locally on an SD card. The inline flow meter is a switch with Hall Effect. It counts the frequency of the switch $(\mathrm{F})$ and the flow is computed as $\mathrm{Q}=\mathrm{F}$ /43 where Q is measured in liters / min. One liter has 5880 output pulses.

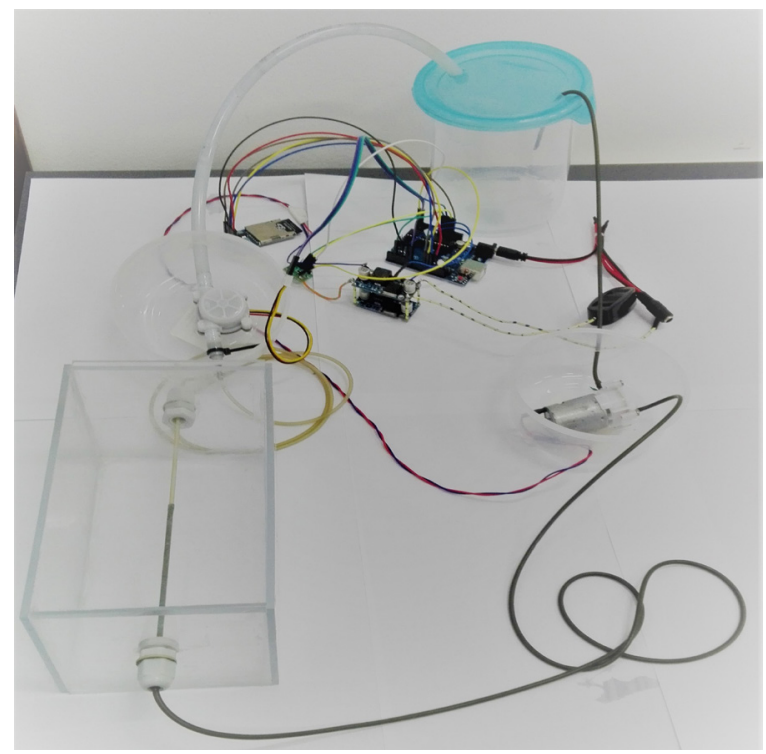

Figure 2. The experimental platform (the vessel phantom on the bottom left side with the flow meter above it, the pump on the right side and the microcontroller)

The micro gear pump works with a voltage between 1-9 V. The great advantage of such 
pumps is that they are simple and cheap, as well as robust with regard to the density of the liquid used. Thus, the use of a viscous, blood like liquid will not affect its functioning.

The pump is controlled by a converter; hence the response time is important. A transit time of under 50 milliseconds was achieved.

The pump is not affected by the fluid temperature variation $\left(15-35^{\circ} \mathrm{C}\right)$ and it can be used as a medical platform due to its small flow rates domain.

For the experiment, the vessel phantom is represented by a silicone tubing of $1.5 \mathrm{~mm}$ interior diameter coupled with another one with $2 \mathrm{~mm}$ as it can seen in Fig. 2.

The flow meter has a measuring range between $0.3-61 / \mathrm{min}$ which makes it suitable for measuring the aorta blood flow and not for the medium arteries or arterioles which have flow rates lower than $300 \mathrm{ml} / \mathrm{min}$. A Buck DC/DC converter is attached in order to better control the power of the pump.

The Atmega microcontroller (through an Arduino development board) sends a Pulse Width Modulation signal to the DC/DC converter with a frequency of $65 \mathrm{KHz}$.

A numerical proportional regulator was implemented in the Arduino platform to adapt the duty cycle according to the flow reference stored as a vector. The regulator has to deal with the delay generated by the travelling of the fluid through the long pipes of the system.

The system is meant to represent a periodic regime that simulates the blood flow dynamics.

Fig. 3 shows the flow measured by means of the flow meter when simulating a periodic blood flow regime.

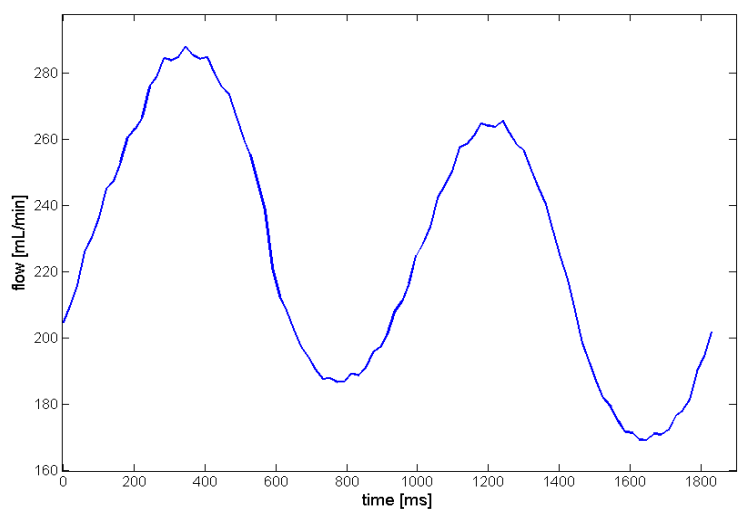

Figure 3. The generated waveform
The flow is designed according to the values presented in the medical literature, showing a clear manifestation of high fluctuations.

\section{Conclusion}

The paper presents an algorithm for 3D reconstruction from two projection views of a vessel centerline by adding the parameter optimisation through minimisation of the 3D reconstruction error.

A detailed validation with the ground truth data acquired from the quantitative X-ray angiography software is given.

Furthermore, an experimental platform for studying the blood flow was designed.

The scope of the research was to present a technical solution which can be easily reproduced with very low costs.

The platform can be used for the evaluation of the blood flow patterns and for medical education.

Further work will include the improvement of the control algorithm by an adaptive robust control (Popescu et al 2017), to better mimic the real coronary artery flow waveforms and to print the 3D model with flexible material.

\section{Acknowledgements}

This work has been funded by University Politehnica of Bucharest, through the "Excellence Research Grants" Program, UPB - GEX 2017. Identifier: UPB GEX2017, Ctr. No. AU 11-1704/2017 DSIS".

\section{REFERENCES}

1. Blondel, C., Malandain, G., Vaillant, R. \& Ayache, N. (2006). Reconstruction of coronary arteries from a single rotational $\mathrm{X}$-ray projection sequence, IEEE Trans. Med. Imag., 25(5), 653-663.

2. Brost, A., Strobel, N., Yatziv, L., Gilson, W., Meyer, B., Hornegger, J., Lewin, J. \& Wacker, F. (2009a). Accuracy of x-ray image-based 3D localization from two C-arm views: a comparison between an ideal system and a real device. In Proc. SPIE, Med. Imag.: Visualization, Image-Guided Procedures, and Modeling, vol. 726. 
3. Brost, A., Strobel, N., Yatziv, L., Gilson, W., Meyer, B., Hornegger, J., Lewin, J. \& Wacker, F. (2009b). Geometric Accuracy of 3-D X-Ray Image-Based Localization from Two C-Arm Views. In MICCAI (pp. 12-19).

4. Bourantas, C. V., Kourtis, I. C., Plissiti, M. E., Fotiadis, D. I., Katsouras, C. S., Papafaklis, M. I. \& Michalis, L. K. (2005). A method for 3D reconstruction of coronary arteries using biplane angiography and intravascular ultrasound images, Comp. Med. Imag. \& Graph., 29, 597-606.

5. Drost S., de Kruif B. J. \& Newport, D. (2018). Arduino control of a pulsatile flow rig, Medical Engineering \& Physics, 51, 67-71.

6. Garcia, J. A., Chen, J., Hansgen, A., Wink, O., Movassaghi, B. \& Messenger, J. C. (2007). Rotational angiography (RA) and three-dimensional imaging (3-DRA): An available clinical tool, Int. J. Cardiovasc. Imag., 23, 9-13.

7. Ioniță, C.N., Mokin, M., Varble, N., Bednarek, D. R., Xiang, J., Snyder, K.V., Siddiqui, A. H., Levy, E. I., Meng, H. \& Rudin, S. (2014). Challenges and limitations of patient-specific vascular phantom fabrication using 3D Polyjet printing. In Proc SPIE Int. Soc Opt Eng., 9038:90380M (pp. 1-23).

8. Marcu, R., Danila, I., Popescu, D., Chenaru, O. \& Ichim, L. (2017). Message Queuing Model for a Healthcare Hybrid Cloud Computing Platform, Studies in Informatics and Control, 26(1), 95-104. doi: 10.24846/ v26ily201711

9. Mechoor, R. R., Schmidt, T. \& Kung, E. (2016). A Real-Time Programmable Pulsatile Flow Pump for In-Vitro Cardiovascular Experimentation, J.Biomech Eng, 138(11), 111002-111002-5.

10. Morar, A., Moldoveanu, F., Moldoveanu, A., Bălan, O. \& Asavei, V. (2017a). GPU Accelerated 2D and 3D Image Processing. In Proc. Federated Conf. on Computer Science and Information Systems, vol. 11 (pp. 653-656).
11. Morar, A., Moldoveanu, F., Petrescu, L., Bălan, O. \& Moldoveanu, A. (2017b). Timeconsistent segmentation of indoor depth video frames. In Transmission and Signal Processing Conf. (pp. 674-677).

12. Petrescu L., Morar, A., Moldoveanu, F., Asavei, V. (2011), Real time reconstruction of volumes from very large datasets using CUDA. In 15th ICSTCC, Sinaia, Romania.

13. Popescu, D., Gliga, L., Dimon, C. \& Olteanu S. (2017). In 14th Int. Conf. on Eng. of Modern Electric Systems (pp. 204-207).

14. Shechter, G. (2004). Respiratory Motion of the Heart: Implications for Magnetic Resonance Coronary Angiography, PhD Thesis. Johns Hopkins University.

15. Sprague, K., Drangova, M., Lehmann, G., Slomka, P., Levin, D., Chow, B. \& deKemp, R. (2006). Coronary X-ray angiographic reconstruction and image orientation, Med. Phys., 33, 707-718.

16. Stefanoiu, D., Borne, P., Popescu, D., Filip, F. G. \& El Kamel, A. (2014). Optimization in Engineering Sciences: Approximate and Metaheuristic Methods: Metaheuristics, Stochastic Methods and Decision Support, 253-308. Wiley-ISTE.

17. Tache, I. A. (2015). Vessels Enhancement in X-ray Angiograms. In IEEE Int. Conf. on $e$-Health and Bioengineering, Iasi, Romania.

18. Tache, I. A. (2017). Patient-specific Hemodynamic Computations: Application to Personalized Diagnosis of Cardiovascular Pathologies, 153-175. Springer International Publishing.

19. Wächter, I. (2009). 3D Reconstruction of Cerebral Blood Flow and Vessel Morphology from X-Ray Rotational Angiography, Phd thesis. UCL.

20. Yang, J., Wang, Y., Liu, Y., Tang, S. \& Chen, W. (2009). Novel Approach for 3D Reconstruction of Coronary Arteries from Two Uncalibrated Angiographic Images, IEEE Trans. on Im. Proces., 18(7), 15631572.

http://www.sic.ici.ro 